\section{PTU-177 RETROSPECTIVE AUDIT OF MANAGEMENT OF PATIENTS ADMITTED TO INTENSIVE CARE UNIT (ITU) WITH SEVERE ACUTE PANCREATITIS(SAP)}

doi:10.1136/gutjnl-2013-304907.267

1."0 Jalil, 'A Iqbal, 'C Patel, 'R Radwan, ${ }^{1} \mathrm{~A}$ Rasheed. ${ }^{1}$ General Surgery, Royal Gwent Hospital, Newport South UK, Newport, UK

Introduction To compare management strategies and mortality of patients admitted to ITU with SAP against national standards and study the group who succumbed to their disease in detail to identify the most accurate prognostic indicators in this group of patients.

Methods Retrospective audit of management and outcome of consecutive patients admitted to ITU with SAP during 2007-2010. The development of necrosis and organ failure(OF)was recorded. Patients were classified into four groups: I (No necrosis or OF), II (sterile necrosis or transient OF), III (infected necrosis or persistent OF) and IV (infected necrosis and persistent OF).

Results Fifty one patients were admitted to ITU with SAP (APACHE II $>8$, modified Glasgow score $>3$ ). All cases fulfilled the Atlanta criteria of SAP. Median age: $66 \pm 17.5$. The overall mortality rate was $38 \%(n-19)$ above national standard of $30 \%$. All 7 patients in group IV died, 5 of them underwent necrosectomy and 1 had CT guided drainage of infected acute fluid collection. The table shows the total number of patients and respective mortality in each group. Neither antibiotics nor nutritional support had significant impact on survival. Outcome (death) correlated with organ dysfunction criteria (Atlanta criteria and APACHE II score).

\begin{tabular}{|c|c|c|c|}
\hline Group & Total Number & Mortality & $\%$ of Mortality \\
\hline 1 & 12 & 0 & $0 \%$ \\
\hline ॥ & 2 & 0 & $0 \%$ \\
\hline III & 30 & 12 & $40 \%$ \\
\hline IV & 7 & 7 & $100 \%$ \\
\hline
\end{tabular}

Conclusion While the presence of infected necrosis or persistent organ failure in SAP (group III) is associated with high mortality, the combination of "infected necrosis and persistent organ failure" (group IV) is uniformly fatal. Further research is necessary to confirm our findings and to explore ways of optimising patients in group III to improve survival.

Disclosure of Interest None Declared.

\section{PTU-178 PITFALLS OF FAECAL ELASTASE ESTIMATION}

doi:10.1136/gutjnl-2013-304907.268

1."R Shah, 'A Varma, 'S Thomson, ' $N$ Direkze. ' Gastroenterology, NHS, Farnborough, UK

Introduction The faecal elastase test measures the concentration of the elastase-3B enzyme found in faecal matter with an enzymelinked immunosorbent assay (ELISA). It is a good indicator of exocrine pancreatic status, and is less invasive and expensive than the secretin-cholecystokinin test, the current gold standard. Levels of faecal elastase lower than $200 \mu \mathrm{g} / \mathrm{g}$ of stool indicate an exocrine pancreatic insufficiency (EPI).

Methods Aim: Faecal elastase is a marker for EPI particularly in chronic pancreatitis and pancreatic malignancy. Our aim was to outline the value of faecal elastase in patients with obscure diarrhoea. Methods: We retrospectively collected data of patients who had a faecal elastase test from June 2010 to 2012. We correlated the abnormal results with symptoms, findings on imaging and response to treatment. We also stratified these findings in patients with ultra low faecal elastase to assess whether the diagnostic yield was different. Paediatric patients were excluded from the study.

Results There were a total of 72 abnormal results out of 523 in this 2 year study of which 33 (45\%) patients had large volume diarrhoea. Of these, $26(78 \%)$ patients had no therapeutic response to pancreatic enzyme supplements (PES) and had normal imaging, 4 had a response and in 3 we could not establish whether PES was trialled. Of the patients who responded to treatment, 2 had imaging suggestive of chronic pancreatitis. 9 of the patients who were investigated for steatorrhoea were on statins but had normal faecal elastase, normal cross sectional imaging and good response to treatment with PES 41 patients had ultra low faecal elastase levels $(<50 \mu \mathrm{g} / \mathrm{g}$ ), of these $32(78 \%)$ patients had significant pancreatic pathology whilst only $9(22 \%)$ had watery diarrhoea.

Conclusion 1- A normal faecal elastase does not exclude EPI - as the 9 patients who were on statins had normal faecal elastase and pancreatic imaging and a dramatic response to PES. Other drugs like azathioprine, steroids causing EPI needs further investigation.

2- An ultra-low faecal elastase level $(<50 \mu \mathrm{g} / \mathrm{g})$, is more likely to be related to significant pancreatic pathology with a response to treatment. Patients with watery diarrhoea are less likely to have a level this low. In these patients stool lyophilising or concentration should be performed before faecal elastase estimation. 3- In our patient group conditions like diabetes, coeliac disease, Addisons disease, gastrointestinal fistulas, short bowel syndrome, microscopic colitis, bile salt malabsorption and previous radiation all lead to falsely low levels without clinical correlation or response to treatment. Faecal elastase in this sub group should be interpreted with caution.

Disclosure of Interest None Declared.

\section{REFERENCE}

The role of faecal elastase-1 in detecting exocrine pancreatic diseaseJohn S. Leeds, Kofi Oppong \& David S. Sanders

\section{PTU-179 DIAGNOSTIC YIELD OF EUS-FNA IN PANCREATIC NEURO-ENDOCRINE TUMOURS (PNET) - SOLID VERSUS CYSTIC PNETS - 9 YEAR EXPERIENCE FROM A TERTIARY CENTRE}

doi:10.1136/qutinl-2013-304907.269

1."V Mitra, 'M Nayar, 'J Leeds, ${ }^{2} \mathrm{~B}$ Haugk, ${ }^{2} \mathrm{~V}$ Wadehra, 'R Charnley, 'B Jaques, 'S White, 'D Manas, 'J French, 'K Oppong. 'HPB; '2Pathology, Freeman Hospital, Newcastle, UK

Introduction The detection and diagnosis of pNETs remains challenging. EUS and EUS-FNA has a significant role in the detection, precise localisation and cytological confirmation of pNETs.

Methods A retrospective review of all pNET patients undergoing EUS-FNA between April 2003 and September 2011 was carried out to determine the efficacy of EUS-FNA in confirming pNETs and compare performance over two consecutive 4 year period.

Results 10 patients ( $3 \%$ of EUS procedures for cystic lesions) with cystic pNETs and 44 (4\% of EUS procedures for solid lesions) with solid pNETs were identified. Table 1 compares the size, demographics and diagnostic performance of radiology, EUS \& cytology in solid and cystic pNETs. 17 and 5 solid and cystic pNETs respectively were diagnosed between 2003 and 2007 while 27 and 5 solid and cystic pNETs were diagnosed between 2008 and 2011. EUS-FNA diagnosis of cystic and solid pNETs has improved from $20 \%$ and $59 \%$ respectively between 2003 and 2007 to $100 \%$ and $81 \%$ respectively between 2008 and 2011. Overall, sensitivity of EUS cytology has improved from $50 \%$ to $84.4 \%(p=0.015)$ during this period. Malignant potential of solid pNETs was higher (54.5\% vs $20 \%$ ) compared to cystic pNETs. Curative resection was higher in patients with cystic pNETs ( $80 \%$ vs $68 \%$ ) compared to solid pNETs. 


\section{Abstract PTU-179 Table 1}

\begin{tabular}{|c|c|c|c|}
\hline & Combined solid \& cystic pNETs $(n=54)$ & Solid pNETs $(n=44)$ & Cystic pNETs $(n=10)$ \\
\hline Sex (Male \%) & AB59 & AB64 & 40 \\
\hline Mean size of lesion in $\mathrm{cm}$ (range) & $2.73(0.7-9.5)$ & $2.95(0.7-9.5)$ & $1.96(0.8-5)$ \\
\hline Sensitivity of CT/MRI in detecting pNET (\%) & $33.3^{1} \AA$ & $38.5^{1} \AA$ & $10^{1}$ \\
\hline Sensitivity of EUS imaging in detecting pNET (\%) & $51.8 *$ & $57 *$ & AB30 \\
\hline $\begin{array}{l}\text { Sensitivity of combined EUS imaging and cytology in } \\
\text { detecting } \mathrm{pNET}(\%)\end{array}$ & $81.5^{* 1}$ & $84.1^{* 1}$ & $70^{1}$ \\
\hline
\end{tabular}

Note: ${ }^{*} p<0.05,{ }^{1} p<0.05, \AA p<0.05$ comparison within columns

Conclusion EUS \& EUS-FNA is a useful test in diagnosing pNETs. Overall, the sensitivity of combined EUS imaging and cytology was significantly better compared to CT/MRI $(p<0.05)$ in detecting pNETs across all groups. Sensitivity of combined EUS imaging and cytology was significantly better compared to EUS imaging alone $(p<0.05)$ in the solid and combined pNET cohort of patients. Sensitivity of EUS cytology was significantly better compared to EUS imaging $(p<0.05)$ in the solid and combined pNET cohort of patients. Comparing the first 4 years to the second, there has been an increase in the number of cases of pNETs and statistically significant improvement in the diagnostic performance of cytology.

Disclosure of Interest None Declared.

\section{Small bowel}

\section{PTU-180 BILE ACID DIARRHOEA - THE GOOD, THE BAD AND EQUIVOCAL RESPONDERS: A TWO CENTRE COMPARISON}

doi:10.1136/gutjnl-2013-304907.270

1,"A Dhaliwal, 'S Chambers, 'C Nwokolo, ${ }^{2} \mathrm{~N}$ Williams, ${ }^{2} \mathrm{~J}$ Cullis, ${ }^{3} \mathrm{P}$ Basumani, ${ }^{4} \mathrm{M}$ Smith, ${ }^{3} \mathrm{~K}$ D Bardhan, ${ }^{1 R}$ Arasaradnam. ' Gastroenterology; ${ }^{2}$ Radiology, University Hospitals of Coventry and Warwickshire NHS Trust, Coventry; ${ }^{3}$ Gastroenterology; ${ }^{4}$ Radiology, Rotherham General Hospital NHS Trust, Rotherham, UK

Introduction Bile acid diarrhoea $(\mathrm{BAD})$ is a common cause of chronic diarrhoea, currently diagnosed using $\mathrm{Se}^{75}$-homo-chloro-tauric-acid (S ${ }^{75} \mathrm{HCAT}$ ) testing, the "gold standard" in the UK and available to centres which support a gamma camera. Although the test has been available for over two decades, uptake has been slow despite its diagnostic value and evidence that treatment with sequestrants can be effective. This stems from a lack of formal clinical trials, which encourages a belief the good results reported may reflect patient selection, resulting in therapeutic nihilism. Thus the aim of this study was to compare treatment results between two centres: experienced $(\alpha)$, using data reported earlier, and novice $(\beta)$, with current data.

Methods The patients recruited comprised those with structural disease (e.g. ileal resection, cholecystectomy), and non-structural, the majority with diarrhoea-predominant irritable bowel syndrome (D-IBS). Abnormal Se ${ }^{75} \mathrm{HCAT}$ results were defined as $<10 \%$ retention on Day-7. Response to treatment: $1^{\text {st }}$ line (cholestyramine, colestid, colesitpol) or $2^{\text {nd }}$ line (colesevelam) was recorded in three categories: good, partial or poor.

Results

Centre $\alpha$ : 2001-2006; recruited $n=162$; treatment assessed $n=129$. Centre $\beta$ : 2008-2012; recruited $n=126$; treatment assessed $n=99$. Response to treatment

\section{Abstract PTU-180 Table}

\begin{tabular}{lll}
\hline Response & Centre $\alpha$ & Centre $\beta$ \\
\hline Good & $60(47 \%)$ & $40(40 \%)$ \\
Partial & $30(23 \%)$ & $23(23 \%)$ \\
Poor & $15(12 \%)$ & $10(10 \%)$ \\
\hline
\end{tabular}

D-IBS - Abnormal Se ${ }^{75}$ HCAT: centre $\alpha 33 \%$, Centre $\beta 31 \%$. Disclosure of Interest None Declared. Conclusion

1. Both centres had similar proportions with abnormal $\mathrm{Se}^{75} \mathrm{HCAT}$ values, suggesting gastroenterologists are able to select appropriate patients who are likely to benefit from the test.

2. Treatment response in both centres was similar, $\sim 70 \%$ responding. This suggests treatment is genuinely effective and not limited only to centres with a special interest.

\section{PTU-181 THE LOW DIAGNOSTIC YIELD OF NON-TARGETED DUODENAL BIOPSIES}

doi:10.1136/gutjnl-2013-304907.271

1."A Young, ${ }^{1} \mathrm{C}$ Robertson, ${ }^{2} \mathrm{~A}$ A Shah, ${ }^{1} \mathrm{M}$ Balsitis. ${ }^{1}$ Pathology; ${ }^{2}$ Medicine, Crosshouse Hospital, Kilmarnock, UK

Introduction Duodenal mucosa of normal endoscopic appearance is often biopsied (non-targeted biopsy) to exclude coeliac disease (CD) as the cause of iron deficiency anaemia or other symptoms. Our aim was to investigate the incidence of diagnosis of CD in nontargeted biopsies, in conjunction with tissue transglutaminase antibody (tTG) test results, in 3 adult age groups.

Methods Review of records of 297 consecutive patients having duodenal biopsy from July to October 2011. Age, indication for endoscopy, endoscopic appearance, tTG status and microscopy findings were recorded.

Results See table. Of 297 total patients, age range was 16-93 years. 60 biopsies (20\%) were from endoscopically abnormal duodenum and $237(80 \%)$ were from endoscopically normal duodenum, the latter including 12 from patients with follow-up of known CD and 7 with other clinical indication to biopsy normal duodenum. Therefore $218(73 \%)$ were non-targeted biopsies with no indication to biopsy normal duodenum other than to rule out CD. 210 (96\%) of the non-targeted biopsies were histologically normal or near normal with no features of CD \& the remaining 8 (4\%) had histological features consistent with CD. All 8 such patients had positive tTG results, 4 before \& 4 after endoscopy, confirming the diagnosis of CD. 60 further patients from the 218 non-targeted group had tTG testing and all were negative. $65(30 \%)$ non-targeted biopsies were from patients aged $\geq 75$ years but $C D$ was not newly diagnosed in this age group. 3 of the 60 patients with endoscopically abnormal duodenum had histological features consistent with a new diagnosis of CD and positive tTG ( $\leq 54$ years $=1,55-74$ years $=2, \geq 75$ years $=0$ ).

Conclusion The majority of duodenal biopsies were non-targeted. CD was identified in $4 \%$ of non-targeted biopsies, in tTG positive patients. Non-targeted biopsies did not show significant findings in tTG negative patients. Reduction in biopsy workload could be achieved if non-targeted biopsies were to be taken only in the setting of positive tTG or other relevant clinical indication; no 